TITLE:

\title{
Power-law behavior in the power spectrum induced by Brownian motion of a domain wall
}

\section{$\operatorname{AUTHOR}(S):$}

Takesue, S; Mitsudo, T; Hayakawa, H

\section{CITATION:}

Takesue, S ...[et al]. Power-law behavior in the power spectrum induced by Brownian motion of a domain wall. Physical Review E 2003, 68(1): 015103.

\section{ISSUE DATE:}

2003-07

URL:

http://hdl.handle.net/2433/50042

RIGHT:

Copyright 2003 American Physical Society 
PHYSICAL REVIEW E 68, 015103(R) (2003)

\title{
Power-law behavior in the power spectrum induced by Brownian motion of a domain wall
}

\author{
Shinji Takesue* and Tetsuya Mitsudo ${ }^{\dagger}$ \\ Faculty of Integrated Human Studies, Kyoto University, Kyoto 606-8501, Japan \\ Hisao Hayakawa ${ }^{\dagger}$ \\ Graduate School of Human and Environmental Studies, Kyoto University, Kyoto 606-8501, Japan
}

(Received 17 March 2003; published 18 July 2003)

\begin{abstract}
We show that Brownian motion of a one-dimensional domain wall in a large but finite system yields a $\omega^{-3 / 2}$ power spectrum. This is successfully applied to the totally asymmetric simple exclusion process with open boundaries. An excellent agreement between our theory and numerical results is obtained in a frequency range where the domain wall motion dominates and the discreteness of the system is not effective.
\end{abstract}

DOI: 10.1103/PhysRevE.68.015103

PACS number(s): 02.50.Ey, 05.40.-a, 89.40.-a

The asymmetric simple exclusion process (ASEP) is one of the simplest models of collective transport of particles $[1-3]$. It can be interpreted as a simplified model of traffic flow [4,5] or as a model of granular flow [6]. Since some exact solutions of ASEP can be obtained under given boundary conditions, we may capture an essential feature of more complicated systems such as traffic flows and granular flows.

In the study of the traffic flow or granular flow, the power spectrum $I(\omega)$ is used to investigate properties of local density fluctuations of vehicles or particles and it commonly shows a power-law form $I(\omega) \sim \omega^{-\alpha}$. For example, in real observation of granular flow, it is confirmed that $\alpha=4 / 3$ [6-8]. On the other hand, the value of the exponent $\alpha$ has not yet been fixed for traffic flows $[9,10]$. It is worth mentioning that the structure factor (Fourier transform of the power spectrum) in the Rouse model, which is the simplest model of polymer dynamics, obeys $\exp \left[-(t / \tau)^{1 / 2}\right]$ with the relaxation time $\tau$ [11]. Namely, the Brownian motion of structured material produces $I(\omega) \sim \omega^{-3 / 2}$.

In this paper, we numerically calculate the power spectrum for the totally ASEP (TASEP), which is the simplest ASEP, and find that this model also exhibits a power law $I(\omega) \sim \omega^{-3 / 2}$. This behavior can be explained by a domain wall theory $[12,13]$.

The TASEP is a continuous-time stochastic process on the one-dimensional lattice which is defined as follows. Consider the set of lattice points $-L / 2,-L / 2+1, \ldots, L / 2$. Each site $i$ is either occupied by a particle $\left(\tau_{i}=1\right)$ or is empty $\left(\tau_{i}=0\right)$. During an infinitesimal time interval $d t$, each particle hops to the right adjacent site with probability $d t$ if the destination site is empty. Moreover, in the case of open boundaries, a particle is injected to site $-L / 2$ with probability $\alpha d t$ if the site is empty, and a particle is extracted from site $L / 2$ with probability $\beta d t$ if the site is occupied.

The steady state of this model is exactly obtained for any parameter values $\alpha$ and $\beta$ and the system size $L$ by the

\footnotetext{
*Present and permanent address: Department of Physics, Kyoto University, Yoshida-Nihonmatsu-Cho, Kyoto 606-8501, Japan.

Electronic address: takesue@phys.h.kyoto-u.ac.jp

${ }^{\dagger}$ Present and permanent address: Department of Physics, Kyoto University, Yoshida-Nihonmatsu-Cho, Kyoto 606-8501, Japan.
}

method of matrix-product ansatz [14], which can also be applied to the more general ASEP $[15,16]$. Moreover, in the steady state, the mean occupation at site $i, \rho=\left\langle\tau_{i}\right\rangle$, and the current $J=\left\langle\tau_{i}\left(1-\tau_{i+1}\right)\right\rangle$ can be calculated in the large- $L$ limit. As a result, it turns out that there exist three phases depending values of $\alpha$ and $\beta$ as follows. When $\alpha<\frac{1}{2}$ and $\alpha<\beta$, the mean occupation is $\rho=\alpha$ and the current is $J$ $=\alpha(1-\alpha)$. This is called the low-density phase. When $\beta$ $<\frac{1}{2}$ and $\beta<\alpha$, the mean occupation is $\rho=1-\beta$ and the current is $J=\beta(1-\beta)$. This is called the high-density phase. When $\alpha>\frac{1}{2}$ and $\beta>\frac{1}{2}$, the mean occupation is $\rho=\frac{1}{2}$, and the current is $J=\frac{1}{4}$. This is called the maximum current phase. The phase diagram is illustrated in Fig. 1.

The thick line in Fig. $1, \alpha=\beta<\frac{1}{2}$, is the coexistence line, where we focus our attention in the following. In this case, there occurs a domain wall between a left-hand region of the low-density phase and a right-hand region of the highdensity phase. It is known that the time-averaged density profile is a linear function of position. This means that the domain wall can be anywhere with equal probability as a result of its random walk. This kind of kink motion can also be obtained from a decouple approximation of ASEP where the equation of density profile is reduced to the inviscid Burgers equation. It should be noted that the inviscid Burgers equation is a model equation of traffic flow [4,9].

The average motion and fluctuations of a domain wall are obtained as follows $[12,13]$. Assume that the system consists of a left domain of particle density $\rho_{-}$and flux $j_{-}$and a right domain of particle density $\rho_{+}$and flux $j_{+}$. Then the drift velocity of the domain wall is derived via the equation of continuity as

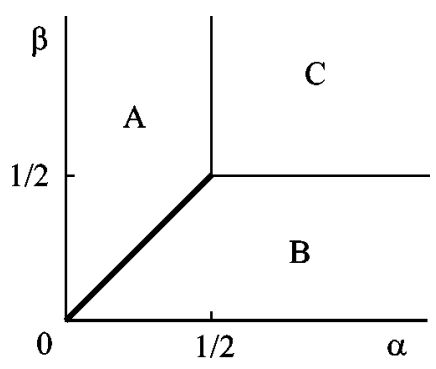

FIG. 1. The phase diagram of the TASEP with open boundaries. Regions $A, B$, and $C$ mean the low-density phase, the high-density phase, and the maximum current phase, respectively. 


$$
V=\frac{j_{+}-j_{-}}{\rho_{+}-\rho_{-}}
$$

This is further interpreted as a result of random walk of the domain wall with hopping rates

$$
D_{+}=\frac{j_{+}}{\rho_{+}-\rho_{-}}, \quad D_{-}=\frac{j_{-}}{\rho_{+}-\rho_{-}},
$$

for a move to the right $\left(D_{+}\right)$and to the left $\left(D_{-}\right)$. In particular, on the coexistence line, the drift velocity vanishes and the diffusion constant for the domain wall motion is given by

$$
D=\frac{\alpha(1-\alpha)}{1-2 \alpha} .
$$

Spatiotemporal patterns of the TASEP clearly indicate the domain wall. See figures in Refs. $[12,16]$. The domain wall picture gives a phenomenological description of the dynamics of the systems. It has been successfully applied for explaining the various features of the system such as the nature of phase transitions and fluctuations in total particle numbers $[12,13]$.

We carried out Monte Carlo simulations of the TASEP with open boundary conditions of system size $L=200$. In our simulations, one Monte Carlo step corresponds to time interval 0.1. Parameter values are chosen to be $\alpha=\beta=0.2$, which are on the coexistence line. After discarding transient parts before a sufficiently large time $T_{0}$, sequences of site values $\left\{x_{t}=\tau_{y}\left(T_{0}+t\right) \mid t=0,1, \ldots, T-1\right\}$ at a fixed position $y$ are recorded. The length of the sequence is $T=2^{20}$. Fourier components of the series are denoted by $\phi_{n}$. That is,

$$
\phi_{n}=\frac{1}{T} \sum_{t=0}^{T-1} x_{t} e^{-i \omega_{n} t}
$$

where $\omega_{n}=2 \pi n / T(n=0,1,2, \ldots$,$) . Then the power spec-$ trum $I(\omega)$ is computed by

$$
I\left(\omega_{n}\right)=T\left\langle\left|\phi_{n}\right|^{2}\right\rangle
$$

where the brackets mean averaging over 256 samples.

Figure 2 shows the simulation result for $y=0$, which lies at the center of the system. It is clearly observed that the power spectrum is proportional to $\omega^{-3 / 2}$ in the frequency range $5 \times 10^{-5} \sim 2 \times 10^{-3}$. In the following, we show that this power-law behavior is induced by Brownian motion of the domain wall.

Now we consider a Brownian motion of a domain wall in an idealized form. Let a domain wall be described by the step function $\theta(x)$ as

$$
\tau(x, t)=\theta(x-X(t))
$$

where $X(t)$ is a Brownian motion in $[-L / 2, L / 2]$. The end points $-L / 2$ and $L / 2$ are assumed to be the reflecting boundaries. Then, the autocorrelation function of $\tau(y, t)$ is given by

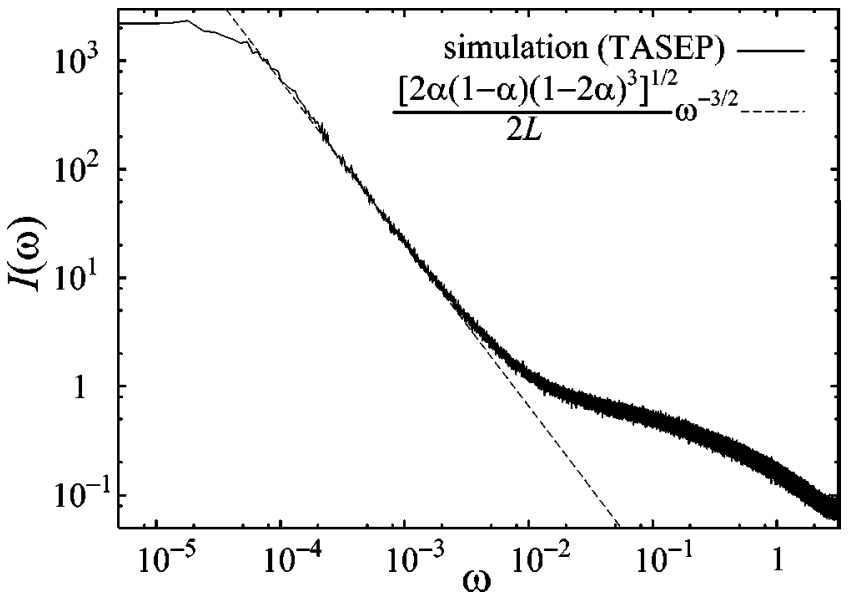

FIG. 2. Power spectrum of time sequence $\left\{\tau_{0}(t)\right\}$ in the TASEP with open boundaries.

$$
\langle\tau(y, t) \tau(y, 0)\rangle=\int_{-L / 2}^{y} d x_{0} \int_{-L / 2}^{y} d x P_{\mathrm{st}}\left(x_{0}\right) P\left(x, t \mid x_{0}, 0\right),
$$

where $P_{\mathrm{st}}(x)$ is the stationary probability density for $X(t)$ $=x$ and $P\left(x, t \mid x_{0}, 0\right)$ is the transition probability density. In other words, if $D$ denotes the diffusion constant for $X(t)$, $P_{\text {st }}(x)$ is the stationary solution of the diffusion equation

$$
\frac{\partial P}{\partial t}=D \frac{\partial^{2} P}{\partial x^{2}}
$$

with the boundary condition

$$
\left.\frac{\partial P}{\partial x}\right|_{x=L / 2}=\left.\frac{\partial P}{\partial x}\right|_{x=-L / 2}=0
$$

and $P\left(x, t \mid x_{0}, 0\right)$ is the solution with initial condition $P\left(x, 0 \mid x_{0}, 0\right)=\delta\left(x-x_{0}\right)$. The equation is easily solved and we find

$$
P_{\text {st }}(x)=\frac{1}{L}
$$

and

$$
\begin{aligned}
P\left(x, t \mid x_{0}, 0\right)= & \frac{1}{L}+\frac{2}{L} \sum_{n: \text { even }} e^{-D \lambda_{n}^{2} t} \cos \lambda_{n} x_{0} \cos \lambda_{n} x \\
& +\frac{2}{L} \sum_{n: \text { odd }} e^{-D \lambda_{n}^{2} t} \sin \lambda_{n} x_{0} \sin \lambda_{n} x
\end{aligned}
$$

where $\lambda_{n}=\pi n / L$ with $n=1,2,3, \ldots$. Then, the integral on the right-hand side of Eq. (7) is carried out to produce

$$
\begin{aligned}
\langle\tau(y, t) \tau(y, 0)\rangle= & \frac{(L+2 y)^{2}}{4 L^{2}}+\sum_{n=1}^{\infty} \frac{e^{-D \lambda_{n}^{2} t}}{L^{2} \lambda_{n}^{2}} \\
& \times\left[1+(-1)^{n-1} \cos 2 \lambda_{n} y\right] .
\end{aligned}
$$


This result can be generalized to negative $t$ by replacing $t$ by $|t|$ in the above expressions.

Because the power spectrum is equal to the Fourier transform of the autocorrelation function, we have

$$
\begin{aligned}
I(\omega) & =\int_{-\infty}^{\infty} e^{-i \omega t}\langle\tau(y, t) \tau(y, 0)\rangle d t \\
& =\frac{\pi}{2} \delta(\omega)+\frac{2 D}{L^{2}} \sum_{n=1}^{\infty} \frac{1+(-1)^{n-1} \cos 2 \lambda_{n} y}{D^{2} \lambda_{n}^{4}+\omega^{2}} .
\end{aligned}
$$

If the oscillatory part including factor $(-1)^{n-1}$ can be ignored and the sum over $n$ can be replaced by an integral, we can evaluate it as

$$
\begin{aligned}
\frac{2 D}{L^{2}} & \sum_{n=1}^{\infty} \frac{1+(-1)^{n-1} \cos 2 \lambda_{n} y}{D^{2} \lambda_{n}^{4}+\omega^{2}} \\
& \simeq \frac{2 D}{\pi L} \int_{0}^{\infty} \frac{d x}{D^{2} x^{4}+\omega^{2}}=\frac{\sqrt{2 D}}{2 L} \omega^{-3 / 2} .
\end{aligned}
$$

Thus, we have arrived at the $\omega^{-3 / 2}$ law. It is remarkable that this result does not depend on position $y$. Actually, as we will see later, $I(\omega)$ shows deviations from the above result in small $\omega$ if $|y|$ is large. This is because the oscillatory part cannot be ignored in the case of large $|y|$ and small $\omega$ even if system size $L$ is large.

Similar calculations can be done if the space is discrete. Assume that a domain wall performs a continuous-time random walk on the one-dimensional lattice between two reflecting walls located at $-L / 2$ and $L / 2$. Denoting hopping rate by $D$, we obtain

$$
\begin{aligned}
I(\omega)= & 2 \pi\left(\frac{[y]+1+\frac{L}{2}}{L+1}\right)^{2} \delta(\omega) \\
& +\frac{2 D}{(L+1)^{2}} \sum_{m=1}^{L} \frac{1+(-1)^{n-1} \cos \left(\frac{m \pi}{L+1}(2[y]+1)\right)}{\omega^{2}+16 D^{2} \sin ^{4}\left(\frac{m \pi}{2(L+1)}\right)},
\end{aligned}
$$

where $[y]$ denotes the largest lattice point that does not exceed $y$. As in the previous case, if the oscillatory part can be ignored, replacement of the sum on the right-hand side by the integral yields for $\omega \neq 0$,

$$
\begin{aligned}
I(\omega) & \simeq \frac{4 D}{\pi(L+1)} \int_{0}^{\pi / 2} \frac{d x}{\omega^{2}+16 D^{2} \sin ^{2} x} \\
& =\frac{\sqrt{2 D} \omega^{-3 / 2}}{2(L+1)}\left(\frac{\frac{\omega}{4 D}+\sqrt{1+\left(\frac{\omega}{4 D}\right)^{2}}}{1+\left(\frac{\omega}{4 D}\right)^{2}}\right)^{1 / 2} .
\end{aligned}
$$

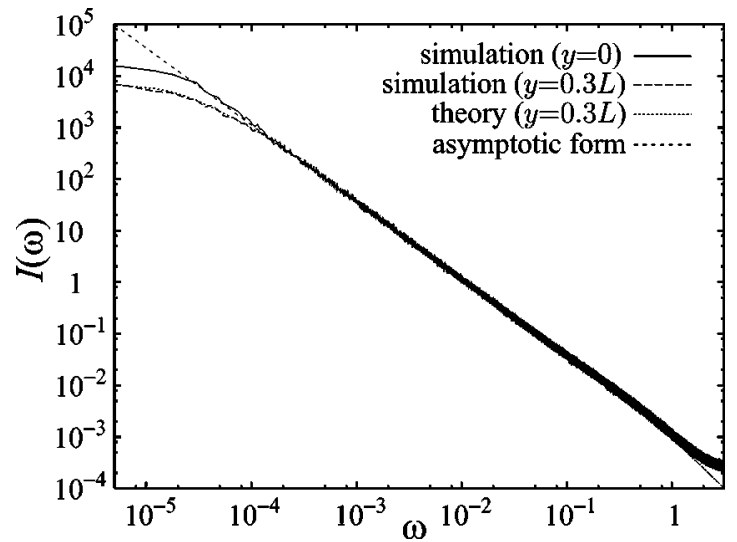

FIG. 3. Power spectra of the time sequences of site values $\{\tau(y, t)=\theta(y-W(t)) \mid t=0,1,2, \ldots\}$ for $y=0$ and $y=0.3 L$, where $W(t)$ means a random walk with hopping rate $D=0.1$. The "theory $(y=0.3 L)$ " means the right-hand side of Eq. (15) and the "asymptotic form" means the right-hand side of Eq. (16).

In the case $\omega \ll 4 D$, this returns to the previous result.

To confirm the above results, we carried out numerical simulations. In the simulation, we deal with a system of length $L=200$ and let a domain wall carry out a random walk $W(t)$ with hopping rate $D=0.1$. Figure 3 shows the numerically obtained power spectra in cases $y=0$ and $y$ $=0.3 L$. In the case $y=0$, the agreement between the theory and the numerical result is excellent. Deviations from the power law are observed only in the very small frequency range $\omega \lesssim \pi^{2} D / L^{2} \simeq 2.5 \times 10^{-5}$, where discreteness of the spectrum $\left\{\lambda_{n}\right\}$ is eminent. In the case $y=0.3 L$, deviations extend to larger frequencies. As mentioned earlier, this is because the oscillatory part cannot be ignored if $|y|$ is large. In any case, however, the power-law behavior is still clearly observed.

Now we return to the TASEP on the coexistence line. Within the domain wall theory, $\tau_{y}(t)$ is approximated by

$$
\tau_{y}(t)=\alpha+(1-\alpha-\beta) \theta(y-X(t)),
$$

where $X(t)$ denotes the position of the domain wall at time $t$. Then, the prefactor of $\omega^{-3 / 2}$ becomes $\sqrt{\alpha(1-\alpha)(1-2 \alpha)^{3} / 2 L^{2}}$. Actually, the straight line in Fig. 2 is depicted with this prefactor. The excellent agreement between the theory and the numerical result confirms that the power-law behavior in the power spectrum is induced by the Brownian motion of a domain wall.

Next, we check system-size dependence of the power spectrum. Figure 4 shows numerically obtained power spectra with various system sizes. As system size $L$ varies, the power-law part is scaled by $L^{-1}$ as expected, while it is not the case in higher frequencies. In fact, the higher-frequency part is independent of system size, representing local fluctuations around the density profile of a step-function shape. Competition between the two parts determines the highfrequency cutoff for the power-law behavior. Because the power law includes factor $L^{-1}$ and the magnitude of the local fluctuations only slightly changes with frequencies, the high-frequency cutoff is nearly proportional to $L^{-2 / 3}$. From 


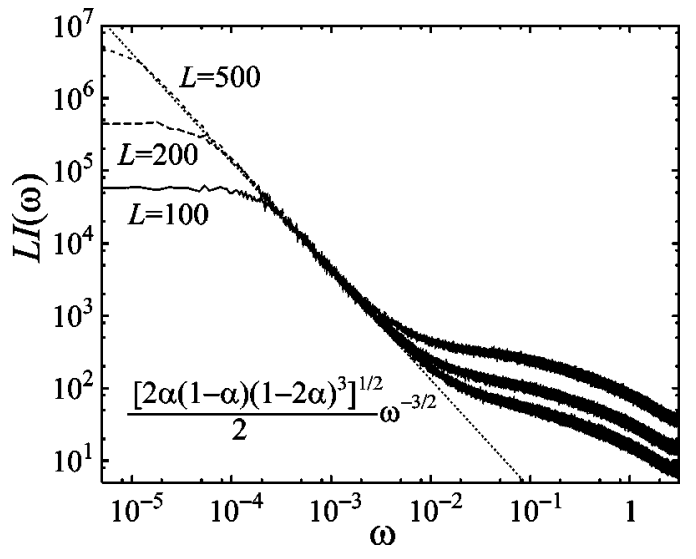

FIG. 4. Power spectra multiplied by system size for the TASEP of various system sizes: $L=100,200$, and 500 .

the numerical data shown in Fig. 4, values of the highfrequency cutoff are estimated as 0.013, 0.009, and 0.0044 for $L=100,200$, and 500, respectively. These values are consistent with the above considerations. On the other hand, the low-frequency cutoff is proportional to $L^{-2}$ as mentioned earlier. Therefore, as the system size is large, the frequency range for the power-law behavior is shifted to lower values and its size increases in the log scale. It is noted that the present power law is a finite-size effect where the boundaries play an important role.

In summary, we have derived that the Brownian motion of a domain wall yields an $\omega^{-3 / 2}$ power spectrum. This mechanism is successfully applied to explain the power law in the power spectrum for local density fluctuations in the TASEP.

It should be noted that the ASEP does not admit more than one domain wall. In real traffic or granular systems, however, many domains can occur and interactions between them may be important. It is a future problem to investigate power spectra in such more complicated systems.

\section{ACKNOWLEDGMENTS}

This work was partially supported by the Hosokawa Powder Technology Foundation and the Inamori Foundation. The authors thank T. Sasamoto for fruitful discussion and useful comments.
[1] H. Spohn, Large Scale Dynamics of Interacting Particles (Springer-Verlag, Berlin, 1991).

[2] B. Derrida, Phys. Rep. 301, 65 (1998).

[3] G.M. Schütz, in Exactly Solvable Models for Many-Body Systems Far From Equilibrium, edited by C. Domb and J.L. Lebowitz, Phase Transitions and Critical Phenomena Vol. 19 (Academic, London, 2001).

[4] D. Helbing, Rev. Mod. Phys. 73, 1067 (2001).

[5] D. Dhowdhury, L. Santen, and A. Schadschneider, Phys. Rep. 329, 199 (2000).

[6] H. Hayakawa and K. Nakanishi, Prog. Theor. Phys. Suppl. 130, 57 (1998).

[7] G. Peng and H.J. Herrmann, Phys. Rev. E 49, 1796 (1994); 51, 1745 (1995).

[8] O. Moriyama, N. Kuroiwa, M. Matsushita, and H. Hayakawa,
Phys. Rev. Lett. 80, 2833 (1998).

[9] T. Musha and H. Higuchi, Jpn. J. Appl. Phys. 15, 1271 (1976); 17, 811 (1978)

[10] S. Tadaki, M. Kikuchi, Y. Sugiyama, and S. Yukawa, J. Phys. Soc. Jpn. 67, 2270 (1998).

[11] P.G. de Gennes, Physics (Long Island City, N.Y.) 3, 37 (1967); M. Doi and S.F. Edwards, The Theory of Polymer Dynamics (Oxford University Press, Oxford, 1986).

[12] A.B. Kolomeisky, G.M. Shütz, E.B. Kolomeisky, and J.P. Straley, J. Phys. A 31, 6911 (1998).

[13] L. Santen and C. Appert, J. Stat. Phys. 106, 187 (2002).

[14] B. Derrida, M.R. Evans, V. Hakim, and V. Pasquier, J. Phys. A 26, 1493 (1993).

[15] T. Sasamoto, J. Phys. A 32, 7109 (1999).

[16] T. Sasamoto, J. Phys. Soc. Jpn. 69, 1055 (2000). 